\title{
Outcomes of robot-assisted radical cystectomy: learning curves, margins and lymph node yield
}

T

hree new studies from an international consortium report the outcomes of robot-assisted radical cystectomy (RARC) for invasive bladder cancer, focusing on the learning curve, surgical margin status, and the rate of lymphadenectomy. While RARC is becoming increasingly widespread among patients with this disease, few data are available on the outcomes of this approach compared with other minimally invasive urologic procedures such as robot-assisted radical prostatectomy.

The International Robotic Cystectomy Consortium (IRCC) prospectively collects data from surgeries conducted at a variety of academic and private centers in the USA and Europe. In total, the three studies analyzed data from up to 527 patients who underwent RARC from 2003 to 2009 . The patients were treated at up to 15 centers, by up to 22 individual surgeons.

The first study aimed to characterize the learning curve of RARC based on data from 496 patients treated by 21 surgeons at 14 institutions. Patients were stratified according to the number of cases performed by the treating surgeon $(<30,30-50$, and $>50)$. Operative time decreased with increasing surgical volume, and lymph node yield increased with increasing volume.

"The most important finding was that acceptable levels of surgical proficiency in RARC could be achieved by the $30^{\text {th }}$ case," observes Matthew Hayn from Roswell Park Cancer Institute, and the lead author on the paper. "This included a positive margin rate of less than $5 \%$, and a lymph node yield of 20 nodes." The investigators plan to augment the IRCC database to incorporate data on operative complications and oncologic outcomes, and ultimately to assess how the learning curve affects cancer-specific and overall survival.

The second paper investigated the incidence of and risk factors for positive surgical margins in an overlapping group of 513 patients from the IRCC database. Overall 35 (6.8\%) had a positive margin on pathologic analysis. On multivariate analysis, increasing age and higher $\mathrm{T}$ stage

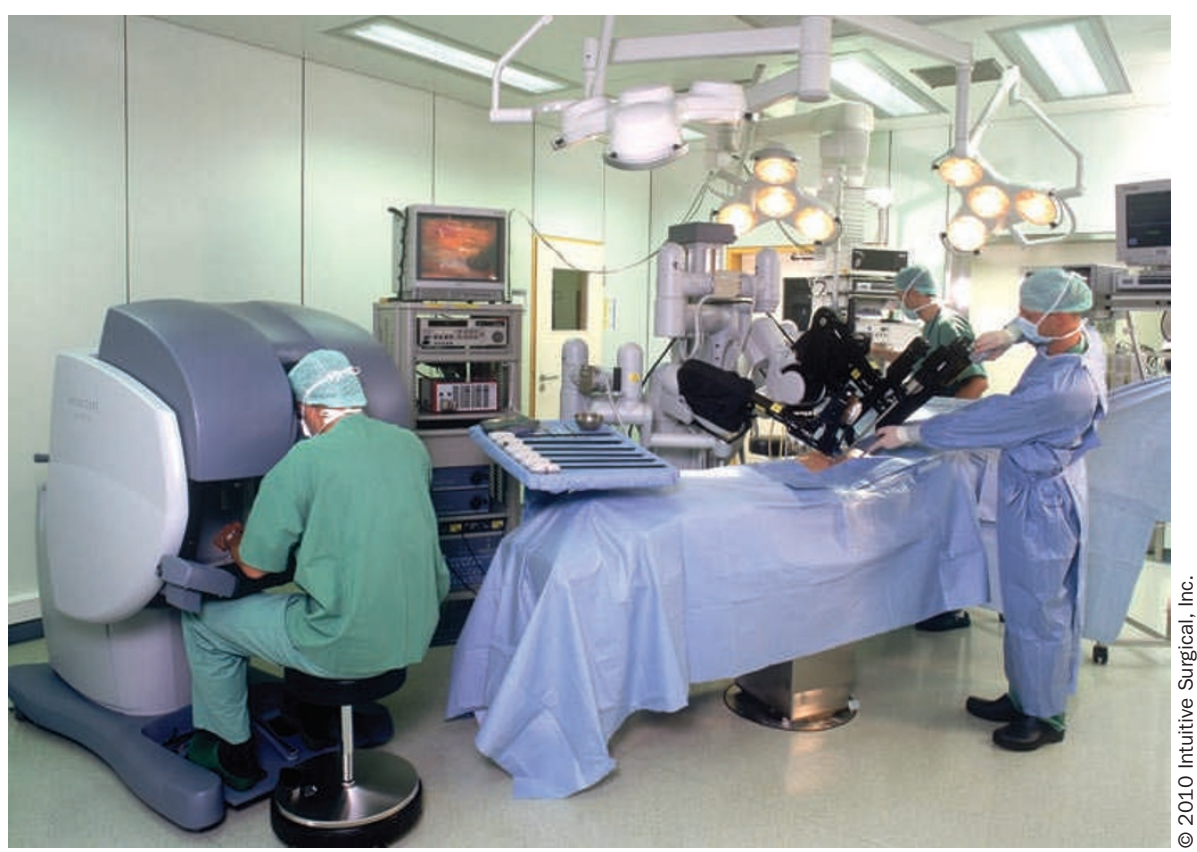

were independent predictors of a positive margin. The learning curve, measured as sequential case number, was not a predictor of margin status. The authors conclude that the positive margin rates for RARC in this series are similar to those for open cystectomy series.

The final study investigated the use of lymphadenectomy, which is both diagnostic and potentially therapeutic, among 527 IRCC-reported patients who underwent RARC. A total of 437 (82.9\%) patients received lymphadenectomydefined as a minimum of 10 nodes removed. On multivariate analysis, pathologic $\mathrm{T}$ stage, sequential case number and surgeon volume were significant predictors of lymphadenectomy. As with positive margins, the authors report that the rates of lymphadenectomy are similar to those of open series, although they highlight the existence of a learning curve for this procedure; in fact, these results are consistent with those of the first study, in which increasing surgical volume was associated with increasing lymph node yield.

Together, these studies report a large RARC series, and provide important outcome data for this procedure. As the collaborative IRCC database matures, valuable information will undoubtedly be revealed about RARC, allowing improved clinical decision-making in patients with locally advanced bladder cancer.

\section{Nick Groves-Kirkby}

\footnotetext{
Original articles Hayn, M. H. et al. The learning curve of robot-assisted radical cystectomy: results from the International Robotic Cystectomy Consortium. Eur. Urol. 58, 197-202 (2010) | Hellenthal, N. J. et al. Surgical margin status after robot assisted radical cystectomy: results from the International Robotic Cystectomy Consortium. Urology 184, 87-91 (2010) | Hellenthal, N. J. et al. Lymphadenectomy at the time of robot-assisted radical cystectomy: results from the International Robotic Cystectomy Consortium. BJU Int. doi:10.1111/ j.1464-410X.2010.09473.x
} 